\title{
Performance Evaluation of AIC Technique for Single and Multiple Primary Bands in OFDM based Cognitive Radios
}

\author{
Gh. Rasool Begh, and Ajaz Hussain Mir
}

\begin{abstract}
Cognitive radio has emerged as an efficient approach to address the problem of spectrum under-utilization. OFDM has been proposed as a potential candidate for the physical layer of cognitive radios due to its inherent characteristics of spectrum shaping, spectrum analysis and robustness to frequency selective fading. One of the major problems of OFDM is high Out Of Band Radiation (OOBR). This is an undesired phenomenon especially if the nearby band is occupied by primary (or licensed) user. Among the proposed techniques to overcome this problem, Active Interference Cancellation (AIC) is considered to be one of the efficient techniques. In this paper, we evaluate the performance of this technique for single and multiple primary bands in OFDM based cognitive radios. For the case of single primary band, it is shown through simulations that narrower primary bands and use of more number of cancellation carriers increases the OOBR reduction in the desired band. For the case of multiple primary bands, the AIC technique is shown to provide better OOBR reduction for larger inter primary band spacing, lesser number of primary bands and more number of cancellation carriers. We also evaluate the bandwidth efficiency for single and multiple primary bands. The bandwidth efficiency shows a linear decrease with the increase in number of cancellation carriers. Multiple primary band system has more bandwidth efficiency degradation than the single primary band system.
\end{abstract}

Keywords-Cognitive Radio, OFDM, Out of Band Radiation, bandwidth efficiency.

\section{INTRODUCTION}

Cognitive radio (CR) allows unlicensed users access to the licensed spectrum, provided they do not cause interference to the licensed primary users (PU) [1], [2]. The unlicensed users are termed as secondary users (SUs) and the licensed users are termed as primary users in the CR context. The secondary users need to sense the spectrum opportunities, called spectrum holes [3], for transmission. These spectrum holes can be used by the secondary users, provided they don't interfere with the primary user transmissions. To avoid interference to the PUs, the SUs should use efficient spectrum shaping techniques. OFDM provides excellent spectrum shaping features, owing to the orthognal properties of its subcarriers [4]. For example, in the simplest case, the subcarriers coinciding with the PU band can be deactivated during SU transmission, to prevent interference to the PU transmissions [4]. However, even after deactivating the subcarriers coinciding with the PU band,

G.R. Begh and A.H. Mir are with the Department of Electronics and Communication Engineering, National Institute of Technology, Srinagar, J\& K, India e-mail:grbegh136@yahoo.com, ahmir@rediffmail.com

Manuscript received March 7, 2016; revised August 20, 2016 appreciable interference power is present in the PU band, due to high Out Of Band Radiation (OOBR) problem of the OFDM [4], [5]. To minimize the OOBR, a number of techniques have been proposed in the literature [6], [7]. These include time windowing [5], [8], Subcarrier Weighting (SW) [9], Active Interference Cancellation (AIC) [10], Adaptive Symbol Transition (AST) [11], Constellation Expansion (CE) [12] and phase adjustment technique [13]. Time windowing and AST provide smooth transition of symbol boundaries to reduce OOBR. These however, suffer from throughput loss due to symbol time extension [5], [11]. In subcarrier weighting technique, all data tones are multiplied by an optimal set of weights to minimize the OOBR. This technique, however, suffers from degradation in the bit error rate (BER) performance due to perturbation in the data tones [9]. Further this method cannot be applied to the OFDM systems using quadrature-amplitude modulation (QAM) since the detection of QAM sysmbols will be severly affected by unknown subcarrier weights [14]. In constellation expansion technique, signal points are mapped to a higher order constellation and the mapped sequence that generates minimum OOBR is chosen for transmission. This however, degrades the error performance at the receiver, as the new signal points are closer to each other than the original signal points [12]. In active interference cancellation (AIC) technique [10], few subcarriers are reserved for transmitting signals to suppress the OOBR in the PU band. These subcarriers are termed as cancellation carriers (CCs) [15], [16]. Using AIC technique, high OOBR reduction of the order of $60-80 \mathrm{~dB}$ can be easily achieved in the band of interest [10]. The OOBR reduction can be increased by increasing the number of cancellation tones, but this leads to a decrease in the number of effective data tones and thereby a decrease in the system throughput. Thus there is a trade off between the OOBR reduction and the bandwidth efficiency in AIC technique. A comparison of various OOBR reduction techniques for multicarrier systems is given in [17]. The authors compare techniques like windowing, AIC, subacrrier weighting, spectral precoding, low pass filtering and filter bank based multi-carrier (FBMC) with conventional OFDM for OOBR reduction. It is shown that FBMC techniques provide the best OOBR reduction (more than $100 \mathrm{~dB}$ ) but at the cost of high computational complexity e.g. For an overlapping factor of 4 , the complexity of FBMC exceeds that of conventional OFDM by a factor of 5 . On the other hand, AIC technique offers much less OOBR reduction (around $40 \mathrm{~dB}$ for a total of 4 cancellation carriers), but exhibits minimum computational 
complexity among the discussed schemes [17]. Thus AIC technique is still of interest due to its low computational complexity.

In this paper, we study the trade off between bandwidth efficiency and OOBR reduction using AIC technique. We study this problem for two different cases: Single primary band cognitive system and multiple primary band cognitive system. We mainly focus on the use of AIC technique for multiple primary bands, which has been less studied in the literature. This paper is organized as follows. In Section 2, system description is given. Simulation results and discussion are presented in Section 3. In Section 4, we draw the conclusions from the study.

\section{System DESCRIPTION}

In this study, we consider a Cognitive radio system that employs OFDM modulation with $\mathrm{N}$ subcarriers. The cognitive engine is assumed to detect the band(s) occupied by the primary users. The secondary users are allowed to share the spectrum with the primary user(s) subject to the condition that the PU communication is not affected. The secondary users use AIC technique to minimize the interference to the detected PU band.

The system model is shown in Fig 1. As depicted in the figure, the input data is mapped to complex constellation stream. It is then divided into $N$ sub streams using serial to parallel converter. The resulting data vector is represented by $\mathbf{X}=\left[X_{0}, X_{1}, X_{2}, . X_{N-1}\right]^{T}$, where $[.]^{T}$ denotes transpose operation. To modulate this data vector on $N$ OFDM subcarriers, an IFFT operation is performed on $\mathbf{X}$. The discrete time OFDM signal obtained at the output of IFFT block is represented by $\mathbf{x}=\left[x_{0}, x_{1}, x_{2}, . x_{N-1}\right]^{T}$ with $x_{n}$ given as

$$
x_{n}=\frac{1}{\sqrt{N}} \sum_{k=0}^{N-1} X_{k} e^{j 2 \pi k n / N}, \quad n=0,1, \ldots, N-1
$$

In matrix form, it can be represented as

$$
\mathbf{x}=F_{(N \times N)}^{*} \mathbf{X}
$$

where $\mathrm{F}$ is $(N \times N)$ Fourier transform matrix, whose $(n, k)^{t h}$ element is given as [11]

$$
F_{n, k}=\frac{1}{\sqrt{N}} e^{-j 2 \pi k n / N}, \quad n, k=0,1, \ldots, N-1
$$

and $(.)^{*}$ is the conjugate operation.

The signal is then extended in time by appending the last $G$ samples of the IFFT output at the beginning of each OFDM symbol. This is termed as cyclic prefix $(\mathrm{CP})$. The output after adding $\mathrm{CP}$ is mathematically represented as

$$
\mathbf{x}^{c p}=F_{((N+G) \times N)}^{*} \mathbf{X}
$$

where $F_{((N+G) \times N)}=\left[\begin{array}{ll}\text { A } & F_{(N \times N)}\end{array}\right]$ with $A$ consisting of last $G$ columns of $F_{(N \times N)}$ [16].

To evaluate the signal spectrum, the time domain signal given in equation (1) is upsampled by a factor of $M$ and the resulting upsampled signal is given by [11]

$$
S=F_{(M N \times(N+G))} \mathbf{x}^{c p}
$$

Using (4), we have

$$
\begin{aligned}
S & =\frac{1}{N} F_{(M N \times(N+G))} F_{((N+G) \times N)}^{*} X \\
& =\mathrm{QX}
\end{aligned}
$$

where

$$
Q=\frac{1}{N} F_{(M N \times(N+G))} F_{((N+G) \times N)}^{*}
$$

Assuming that a PU band with a width of $W$ subcarriers is detected within the OFDM band by the cognitive engine, the SU deactivates the subcarriers corresponding to the detected band and also reserves $c$ cancellation subcarriers (CCs) on the edges of the detected PU band. The interference caused by the data subcarriers in the PU band is given by [11

$$
I_{P}=Q_{W} X_{W}
$$

where $Q_{W}$ consists of only those rows of $Q$ that correspond to the PU band and $X_{W}$ is same as $X$ with the subcarriers coinciding with the PU band and the $c$ cancellation subcarriers turned OFF. The interference power given in equation (8) needs to be minimized by transmitting cancellation tones over $c$ subcarriers on the edges of the PU band. If $h$ is the cancellation tone vector of length $2 c$, then total interference power in the PU band is

$$
P_{p}=\left\|Q_{I} h+I_{P}\right\|^{2}
$$

where $Q_{I}$ consists of only those columns of $Q_{W}$ that correspond to the cancellation subcarriers.

The total interference power in equation (9) needs to be minimized and as such forms a linear least squares problem. The solution of equation (9) given in [10] is

$$
h=-\left(Q_{I}^{H} Q_{I}\right)^{-1} Q_{I}^{H} I_{P}
$$

where $(.)^{H}$ denotes conjugate transpose operation.

Using (8) we get

$$
h=T_{I} X_{W}
$$

where $T_{I}=-\left(Q_{I}^{H} Q_{I}\right)^{-1} Q_{I}^{H} Q_{W}$.

To observe the effect of cancellation carriers on OOBR reduction, we simulate an OFDM based CR system with 128 subcarriers using BPSK symbols. The primary band is assumed to span over a width of 16 subcarriers from subcarrier \#61. The weights of CCs are computed using equation 10 without any constraint. The OOBR reduction achieved using one, two and three CCs is shown in Fig. 2. For comparison, the OOBR reduction achieved using conventional OFDM technique, is also shown in the figure. It is observed from the figure that a reduction of around $35 \mathrm{~dB}$ is achieved using single cancellation carrier (CC) and around $65 \mathrm{~dB}$ is achieved for three $\mathrm{CC}$ s case. This is quite appreciable in comparison to $20 \mathrm{~dB}$ achieved in carrier nulling technique (i.e. deactivating the subcarriers in the PU band). However, as depicted in Fig. 2 . computing cancellation carrier weights as an unconstrained problem, results in spectrum overshoot (e.g. around $20 \mathrm{~dB}$ for three CCs) in the vicinity of the PU band. To overcome this problem, the complex weights of the cancellation carriers can be computed under an upper power constraint. This leads to the following constrained optimization problem:

$$
h^{o p}=\arg \min _{h}\left\|Q_{I} h+I_{P}\right\|^{2},
$$




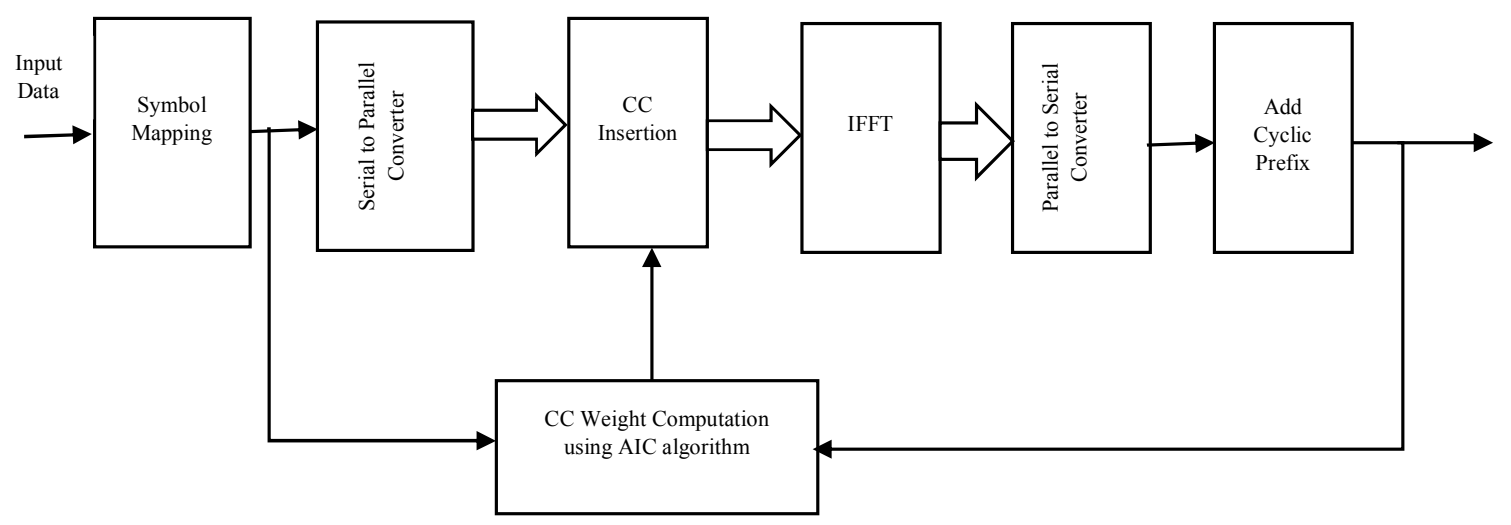

Fig. 1. Block diagram of OFDM transmitter using AIC technique for OOBR reduction

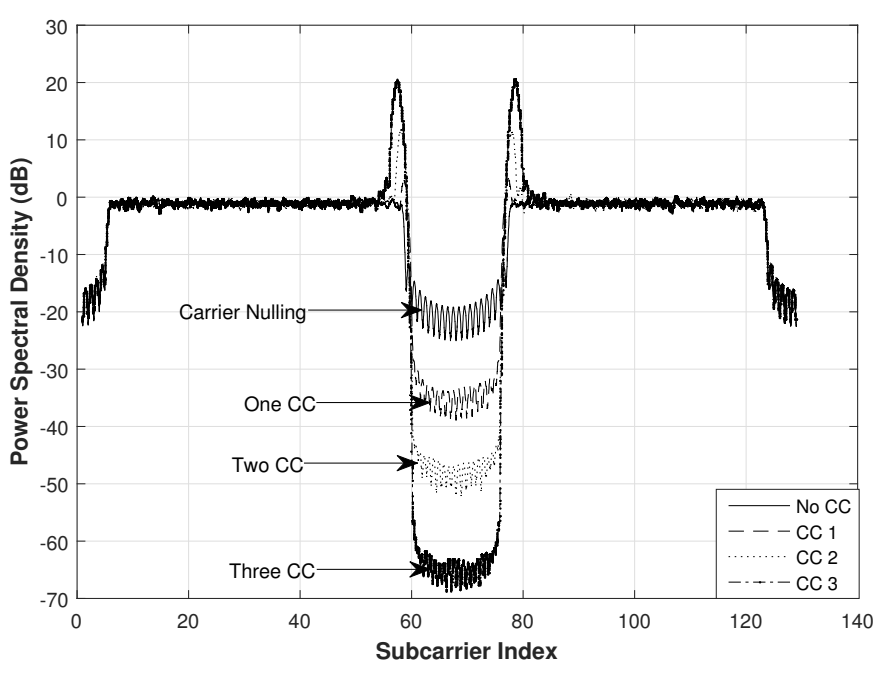

Fig. 2. OOBR Reduction using AIC technique (Unconstrained Case)

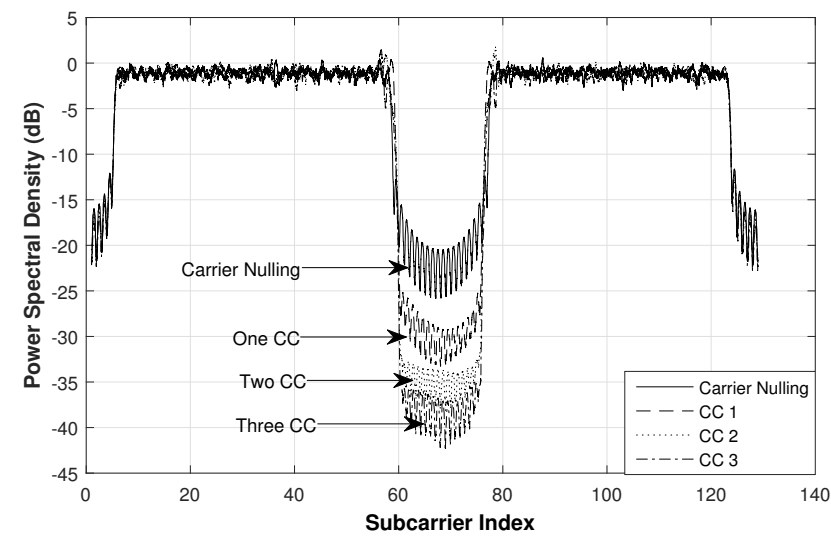

Fig. 3. OOBR Reduction using AIC technique (Constrained Case)

$$
\text { s.t. }\left|h_{i}\right|^{2} \leq \alpha, \quad i=0,1, \ldots, 2 c
$$

where $h^{o p}$ represents the optimal weight vector for cancellation subcarriers with a length of $2 c$ and $\alpha=E\left|h_{i}\right|^{2}, i=$ $1,2, \ldots N$, is the power constraint on cancellation carriers and $E$ represents the expectation operation. Figure 3 shows that by computing optimal weights for the cancellation tone vector, we can overcome the problem of spectrum overshoot but the overall Out Of Band Radiation (OOBR) reduction decreases in comparison to the unconstrained case of AIC technique. However, this technique still offers better reduction than the existing techniques like Adaptive symbol transition [11], Phase adjustment technique [13], windowing techniques [5], [8] and subcarrier weighting [9]. The high OOBR reduction achieved using the AIC technique forms the motivation for our study. We have considered the following two scenarios in this paper:

- Single PU band within OFDM band

- Multiple narrow PU bands within OFDM band

These scenarios are discussed in further sections of this paper.

\section{A. Single PU band}

In the single PU band case, a primary user is detected by the cognitive receiver and the secondary user (SU) refrains from transmitting in that band. To reduce the interference caused by the data subcarriers of the secondary user to the PU band, the secondary user uses AIC technique and reserves some subcarriers for interference cancellation. The amount of cancellation depends on the number of reserved subcarriers. However, increasing the number of cancellation subcarriers reduces the number of effective data subcarriers and hence reduces the bandwidth efficiency. Thus there is a trade off between OOBR reduction and bandwidth efficiency. In section III-A of the paper, we quantify this effect through simulations.

\section{B. Multiple PU bands}

In this scenario, we assume multiple narrow bands that are relatively small in comparison to the total available bandwidth of the cognitive system. This scenario is of interest, when the bandwidth of primary user is very small in comparison to the total bandwidth of the cognitive system e.g. A cognitive system can use upto three consecutive channels $(18 \mathrm{MHz})$ in IEEE 802.22 standard [16]. In comparison to this cognitive system, a wireless microphone occupying a bandwidth of 200 $\mathrm{kHz}$ is a narrowband system. According to FCC regulations, wireless microphones can operate in both unlicensed and licensed modes [18]. When operating in licensed mode, these devices need to be protected from secondary user's transmissions. Since multiple wireless microphones can appear within 
single SU band, the scenario is termed as multiple PU band scenario. To the best of our knowledge, multiple narrow PU bands scenario has not been explored much in the literature. In multiple narrow PU bands, we need to minimize interference caused by active data carriers at multiple band locations. Since in AIC technique, each PU band needs cancellation carriers at its edges, so for $M$ primary bands within OFDM spectrum, we need $M * 2 * c$ cancellation carriers, where $c$ is the number of cancellation carriers (CCs) used on each PU band edge. The effective number of data carriers available for SU's transmission, therefore reduces to $N-M * 2 * c-\sum_{i=1}^{M}\left(N_{i}\right)$ in comparison to $N-2 * c-N_{P}$ for single PU band case, where $N_{P}$ denotes the number of subcarriers corresponding to the bandwidth occupied by PU in single band case and $N_{i}$ denotes the number of subcarriers corresponding to the bandwidth occupied by $i^{\text {th }}$ PU band. This in turn reduces the bandwidth efficiency of the cognitive system. To quantify the effect of number of CC's on bandwidth efficiency and interference reduction, simulations are performed in MATLAB. The results of these simulations are discussed in the next section.

\section{SIMULATION RESULTS AND DISCUSSION}

In this paper, we study the performance of Active Interference Cancellation (AIC) Technique through simulations performed in MATLAB. We assume an OFDM based Cognitive radio with 512 subcarriers. A cyclic prefix of length 64 is added to each OFDM symbol. The data subcarriers are modulated by BPSK symbols and the subcarriers in PU band(s) are deactivated. Thus the power spectral density observed in the PU band, is only due to other subcarriers and is a measure of Out Of Band Radiation (OOBR). This is termed as interference level / interference power in our study. An upsampling factor of 16 is used to find the spectrum in the PU band. The performance of the AIC technique is studied in two different scenarios.

\section{A. Single PU band case}

We consider three different widths spanning over 8,16 and 32 subcarriers, for single PU band detected within the OFDM band. The effectiveness of AIC technique in reducing the OOBR is tested by increasing the number of cancellation carriers from one to 15 on either side of the PU band. Fig. 4 shows the variation of interference level in the PU band with the number of cancellation carriers used, for different widths of the PU band. It is clear from Fig. 4 that the interference level in the PU band decreases with increase in number of cancellation carriers. However, increasing number of cancellation carriers leads to a decrease in the number of effective data carriers and hence decreases the throughput / bandwidth efficiency, thereby defeating the main objective of cognitive radios. It can also be observed from Fig. 4 that there is more reduction in narrow PU band than a wider one, if the number of cancellation carriers used is more than five. This difference is much larger for higher number of CCs used (say ten or more). This difference is attributed to the fact that the optimized weights of cancellation carriers are computed under a power constraint to overcome the spectrum overshoot near

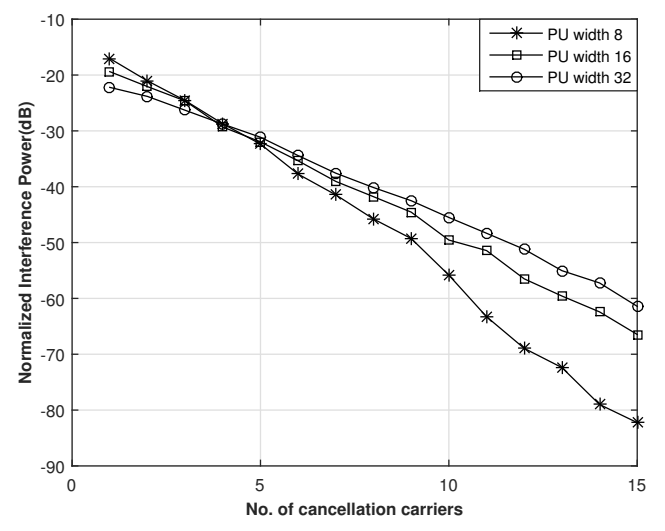

Fig. 4. Effect of number of cancellation carriers and the width of PU band on Interference power in single PU band.

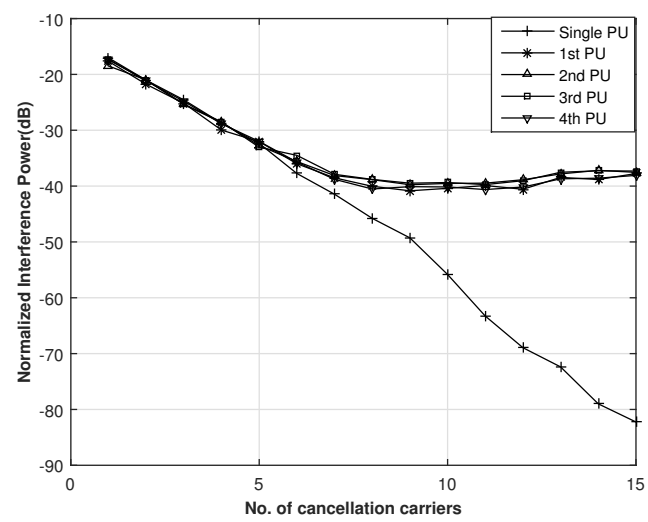

Fig. 5. Effect of number of cancellation carriers on Interference power in multiple PU bands.

the PU band edges ( as discussed in Section 2). Thus for a fixed number of cancellation carriers, interference cancellation can take place in a better manner for a narrower PU band than a wider one.

\section{B. Multiple PU bands case}

In this case, we first consider 4 primary bands, separated equally from each other, and each of them spanning over a width of 8 subcarriers. Thus the total width of the multiple bands is equal to the single primary band of Section III-A All other parameters are kept same as in single PU band case. Fig. 5 depicts a comparison of interference levels of a single PU band and each of the four multiple PU bands. It is clear that interference level in each of the multiple PU band is almost same and is thus independent of the position of detected PU band. However, the reduction in multiple PU bands is less in comparison to single PU band case especially for larger number of cancellation carriers $(\mathrm{CC}>10$ ). This is attributed to the fact that increasing the number of cancellation carriers brings the $\mathrm{CC}$ of one PU band closer to the other PU band thereby hindering its interference cancellation capability. Further it is also observed from Fig. 5 that there is almost no improvement in interference reduction for multiple PU band case as the number of CCs is increased beyond 7 . 


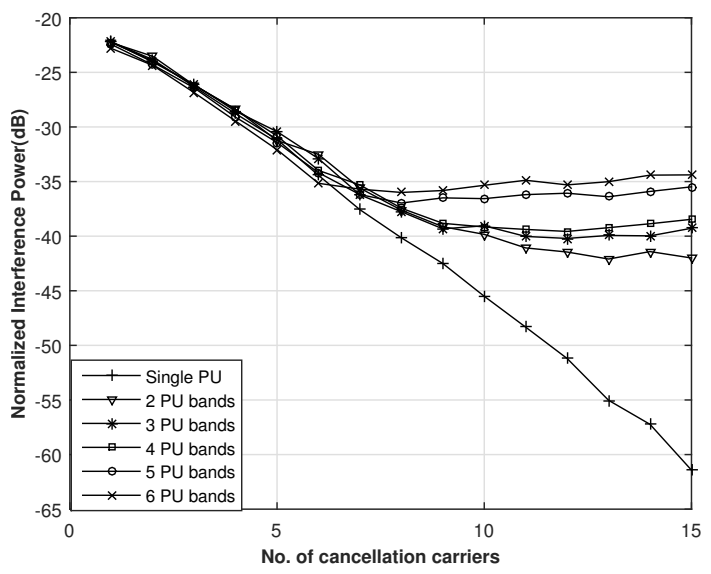

Fig. 6. Effect of number of PU bands on Interference power in multiple PU bands.

1) Effect of increasing number of PU bands: In this case, we see the variation in interference power by changing the number of detected PU bands within the OFDM spectrum. We compare the interference reduction as the number of PU bands is varied from 2 to 6 . Each of these bands spans over a width of 32 subcarriers. Fig. 6 depicts a comparison of average interference power as the number PU bands is increased from 2 to 6 . It is clear that the reduction of interference power in the detected PU bands decreases as the number of PU bands within OFDM spectrum increases. Thus interference cancellation behavior of AIC technique shows a decreasing trend as the number of detected PU bands increases from one to multiple PU bands. This is attributed to the fact that, increasing number of PU bands, reduces inter-PU band spacing and hence $\mathrm{CC}$ signals transmitted for one PU band may interfere with the other band, thereby increasing OOBR in that band.

2) Effect of inter PU band spacing: In this case, we simulate the effect of changing spacing between the detected PU bands. We consider three PU bands each of width 8 subcarriers detected within the OFDM spectrum. In this study, we have taken three inter PU band spacings-100, 75 and 50 subcarrier spacings. Effect of inter PU band spacing on interference reduction is shown in Fig. 7. It is clear from the figure that the interference reduction behaviour of AIC technique shows an upward trend as the spacing between PU bands is decreased. This is more pronounced for larger number of CCs. This can again be attributed to the fact that signals carried by $\mathrm{CCs}$ for one PU band cause interference to the other nearby PU bands. Thus as the inter PU band spacing is reduced, there is less suppression in the OOBR.

\section{Bandwidth Efficiency}

AIC technique uses $c$ cancellation carriers on either side of PU band. Assuming there are $M$ narrow PU bands present in the OFDM spectrum of $B \mathrm{~Hz}$, the number of effective data carriers is $N-2 M * c$. In contrast to the discussion given in section II-B, we include the subcarriers coinciding with the PU band(s), because these subcarriers are also used for data

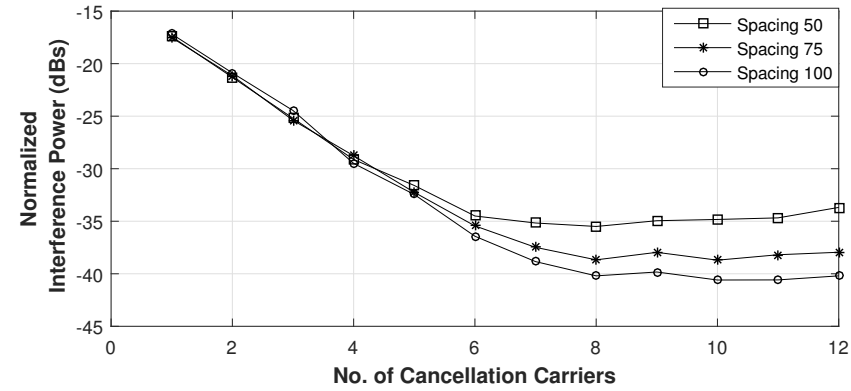

Fig. 7. Effect of inter PU band spacing on Interference power in multiple PU bands.

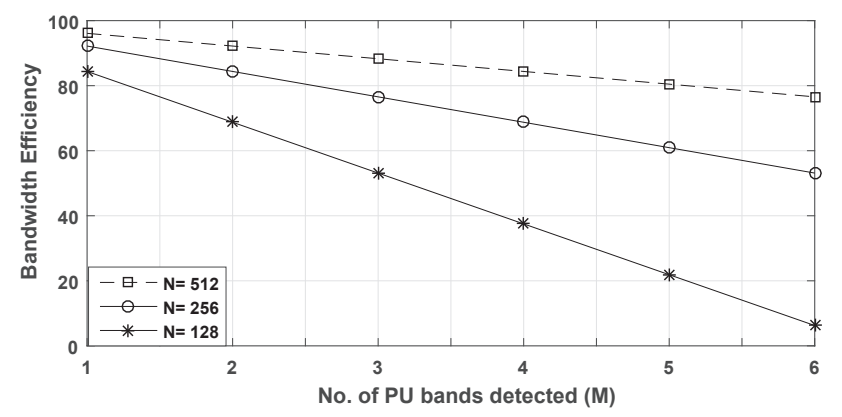

Fig. 8. Effect of number of PU bands, CCs and no. of subcarriers on bandwidth efficiency.

transmission. Thus the Useful Bandwidth for transmission is given by

$$
B_{u}=(N-2 * M * c) * \frac{B}{N}
$$

where $B / N$ is bandwidth per subcarrier. Therefore bandwidth efficiency is given by

$$
\eta_{b}=\frac{\text { Useful Bandwidth }}{\text { Total Available Bandwidth }}
$$

Using equation (13)

$$
\eta_{b}=\left\{1-\frac{(2 * M * c)}{N}\right\}
$$

It is clear from Eqn (14) that the bandwidth efficiency depends on the number of PU bands, cancellation carriers and the number of subcarriers in the OFDM band. In the simulations, we fixed the number of CCs on either edge of PU band as 10 . A plot of bandwidth efficiency for different values of PU bands (M) and subcarriers is shown in Fig. 8 It is clear from the Fig. 8 that there is a linear decrease in bandwidth efficiency with the number of detected PU bands. We have computed the results for three values of $N$ i.e. $128,256 \& 512$. It is clear from Fig. 8 that for a single PU band (i.e. $M=1$ ), there is not appreciable increase in bandwidth efficiency as the value of $N$ is increased from 128 to 512. Since the higher values of $N$ increase the signal processing, it is better to use lower values of $N$ (say $N=128$ in our case), for single PU band. For multiple PU band case (i.e. $M>1$ ) there is an appreciable increase in bandwidth efficiency in comparison to single band case $(M=1)$, especially for higher values of 
$M$. For example, in our simulation scenario, the bandwidth efficiency increases by about $12 \%$ and $34 \%$ for $M=1 \&$ $M=2$ cases respectively, as $N$ is increased from 128 to 512 . This is depicted in Fig. 8 Thus for multiple PU band case, it is better to use more number of subcarriers (e.g. $N=512$ ) to increase the bandwidth efficiency. This however, requires more signal processing and adds to the system complexity.

\section{CONCLUSION}

OFDM based Cognitive radios suffer from high Out Of Band Radiation (OOBR) problem. Active interference cancellation (AIC) technique based on cancellation carriers, is one of the popular techniques to reduce OOBR. In this paper, we study the performance of this technique for single wide primary user band and multiple narrow primary user bands. For single wide primary band, the performance is compared for the cases of unconstrained and constrained computation of cancellation carrier weights. It is seen that, unconstrained computation of the weights leads to large OOBR reduction in the primary band. However, it causes spectrum overshoots in the vicinity of primary user band. A reduction of about $60 \mathrm{~dB}$ with an overshoot of about $20 \mathrm{~dB}$ is obtained for three cancellation carriers on either side of PU band. Constrained computation of these weights, although removes the spectrum overshoot problem but offers much lesser reduction of about $40 \mathrm{~dB}$ for three cancellation carriers. Further, for single primary user band, the reduction increases with the increase in number of cancellation carriers. This however, reduces the bandwidth efficiency as the cancellation carriers do not transmit any useful data. It is also seen that OOBR reduction in wider primary bands is less in comparison to narrow primary bands for the same number of cancellation carriers. In multiple primary user bands, it is seen that much lesser reduction is achieved in comparison to the single band case for the same number of cancellation carriers. We study the effect of inter primary band spacing and the number of detected primary bands on the OOBR reduction and on the bandwidth efficiency. It is seen that there is a decrease in OOBR reduction, if the detected primary bands are closer to each other. Further it is observed that, larger the number of detected primary bands in the OFDM band, lesser is the OOBR reduction. This deterioration in performance of AIC technique for multiband case occurs due to closeness of cancellation carriers to primary bands other than their desired band. It is also observed that, there is a decrease in bandwidth efficiency with the increase in number of primary bands and with the increase in number of cancellation carriers. However, increasing the number of subcarriers in the given OFDM band has a positive effect on the bandwidth efficiency. Thus it can be used to compensate for the bandwidth efficiency loss in case of large number of primary bands but at the cost of more signal processing.

\section{REFERENCES}

[1] I. F. Akyildiz, W.-Y. Lee, M. C. Vuran, and S. Mohanty, "Next generation/dynamic spectrum access/cognitive radio wireless networks: a survey," Computer Networks, vol. 50, no. 13, pp. 2127-2159, 2006.

[2] A. Goldsmith, Wireless communications. Cambridge university press, 2005.
[3] S. Haykin, "Cognitive radio: brain-empowered wireless communications," Selected Areas in Communications, IEEE Journal on, vol. 23, no. 2, pp. 201-220, 2005.

[4] H. Mahmoud, T. Yücek, H. Arslan et al., "OFDM for cognitive radio: merits and challenges," Wireless Communications, IEEE, vol. 16, no. 2, pp. 6-15, 2009.

[5] T. Weiss, J. Hillenbrand, A. Krohn, and F. Jondral, "Mutual interference in ofdm-based spectrum pooling systems," in Vehicular Technology Conference, 2004. VTC 2004-Spring. 2004 IEEE 59th, vol. 4, May 2004, pp. 1873-1877 Vol.4.

[6] Z. You, J. Fang, and I.-T. Lu, "Out-of-band emission suppression techniques based on a generalized ofdm framework," EURASIP Journal on Advances in Signal Processing, vol. 2014, no. 1, pp. 1-14, 2014.

[7] X. Huang, J. A. Zhang, and Y. J. Guo, "Out-of-band emission reduction and a unified framework for precoded ofdm," IEEE Communications Magazine, vol. 53, no. 6, pp. 151-159, June 2015.

[8] I. Budiarjo, H. Nikookar, and L. P. Ligthart, "Cognitive radio modulation techniques," Signal Processing Magazine, IEEE, vol. 25, no. 6, pp. 24 34, 2008.

[9] I. Cosovic, S. Brandes, and M. Schnell, "Subcarrier weighting: a method for sidelobe suppression in ofdm systems," Communications Letters, IEEE, vol. 10, no. 6, pp. 444-446, 2006.

[10] H. Yamaguchi, "Active interference cancellation technique for MBOFDM cognitive radio," in Microwave Conference, 2004. 34th European, vol. 2. IEEE, 2004, pp. 1105-1108.

[11] H. Mahmoud, H. Arslan et al., "Sidelobe suppression in OFDM-based spectrum sharing systems using adaptive symbol transition," Communications Letters, IEEE, vol. 12, no. 2, pp. 133-135, 2008.

[12] S. Pagadarai, R. Rajbanshi, A. M. Wyglinski, and G. J. Minden, "Sidelobe suppression for ofdm-based cognitive radios using constellation expansion," in Wireless Communications and Networking Conference, 2008. WCNC 2008. IEEE. IEEE, 2008, pp. 888-893.

[13] E. H. M. Alian and P. Mitran, "A phase adjustment approach for interference reduction in ofdm-based cognitive radios," Wireless Communications, IEEE Transactions on, vol. 12, no. 9, pp. 4668-4679, 2013.

[14] D. Qu, Z. Wang, and T. Jiang, "Extended active interference cancellation for sidelobe suppression in cognitive radio ofdm systems with cyclic prefix," Vehicular Technology, IEEE Transactions on, vol. 59, no. 4, pp. 1689-1695, 2010.

[15] S. Brandes, I. Cosovic, and M. Schnell, "Reduction of out-of-band radiation in ofdm systems by insertion of cancellation carriers," Communications Letters, IEEE, vol. 10, no. 6, pp. 420-422, 2006.

[16] E. H. M. Alian, H. E. Saffar, and P. Mitran, "Cross-band interference reduction trade-offs in siso and miso ofdm-based cognitive radios," Wireless Communications, IEEE Transactions on, vol. 11, no. 7, pp. 2436-2445, 2012.

[17] W. Jiang and M. Schellmann, "Suppressing the out-of-band power radiation in multi-carrier systems: A comparative study," in Global Communications Conference (GLOBECOM), 2012 IEEE. IEEE, 2012, pp. 1477-1482.

[18] "Wireless michrophones." [Online]. Available: https://www.fcc.gov/ general/wireless-microphones-0

Gh. Rasool Begh has done Bachelor of Engineering (B.E) in Electronics \& Communication Engineering and $\mathrm{M}$. Tech (Communication and Information Technology) from National Institute of Technology Srinagar (J \& K), India. $\mathrm{He}$ is working as Assistant Professor in the Department of E \& C.E at NIT Srinagar. He has guided a number of $\mathrm{M}$. Tech thesis related to OFDM, Cognitive Radios, WLANs and Security. Presently he is pursuing Ph. D in National Institute of Technology Srinagar. His areas of interest include Cognitive Radios, OFDM, MIMO and Security. 
Ajaz H Mir has done his Bachelor of Engineering (B.E.) in Electrica Engineering with specialization in Electronics and Communication Engineering from REC Srinagar (J\& K) India in 1982. He did his M. Tech in Computer Technology and $\mathrm{Ph}$. D both from IIT Delhi in the year 1989 and 1996 respectively. He was Chief Investigator of Ministry of Communication and Information Technology, Govt. of India project: Information Security Education and Awareness (ISEA). He has been guiding $\mathrm{PhD}$ and M. Tech. thesis related to the area of Security and other related areas. He has a number of International publications to his credit. Presently he is working as Professor in the Department of Electronics and Communication Engineering and Dean Faculty at NIT Srinagar, Kashmir, India. His areas of interest are Biometrics, Image Processing, Security, Wireless Communication and Networks 